\title{
Whole-mount Dual Labeling of Regenerating Bone and Vasculature in Axolotl Appendages
}

\author{
Lauren DiMartino $^{1}$, Rodrigo Montoro ${ }^{1}$, Renee Dickie $^{1}$ \\ ${ }^{1}$ Department of Biological Sciences, Towson University, Towson, MD, USA
}

Bone is a highly vascular tissue and angiogenesis, the formation of new capillaries from pre-existing vessels, plays a pivotal role in skeletal growth. Therapies that increase angiogenesis have been shown to improve bone repair (e.g., [1]). The coupling of blood vessel and bone formation has received wide attention in mammalian models of bone healing (e.g., [2]), but little is known of the interrelationship between angiogenesis in osteogenesis within profoundly regenerative environments. In particular, these data are lacking for the pre-eminent model of vertebrate regeneration, the axolotl (Ambystoma mexicanum). This salamander, unlike the more widely employed zebrafish model, is capable of extensive, repeated, endochondral bone regrowth throughout its lifespan. Genetic tools facilitating imaging of these animals, however, are as yet extremely limited compared to those available for zebrafish. This necessitates the development of other tissue labeling techniques. To develop a methodological foundation for investigating the role of angiogenesis in osteogenesis within the context of endochondral bone regeneration, we have combined and adapted tissue preparation methods more widely used in other vertebrate models to permit simultaneous whole mount imaging of the regenerating vasculature and bone in axolotls. We have previously [3] developed perfusion-based methods for labeling the vasculature within regenerating axolotl tissue. In the present work, we sought to determine if the specimen processing required for bone labeling was compatible with this vascular marking technique. Vascular perfusion combined with whole mount labeling allows us to examine the structures of interest in the regenerating appendage within a three dimensional context. The methods provide an inexpensive, straightforward means for simultaneous imaging of the microvasculature and bone using light microscopy.

Adult white strain axolotls (purchased as sub-adults from the Ambystoma Genetic Stock Center, RRID:AGSC_101S) were anesthetized by immersion in benzocaine diluted in Holtfreter's solution prior to measurement and tail amputation. Alcohol-cleaned surgical carbon steel industrial razor blades were used to amputate the distal $25 \%$ of the total tail length. Following amputation, a subset of animals was euthanized weekly by immersion in an overdose of tricaine. All procedures were in accordance with an approved animal protocol overseen by the University's Animal Care and Use Committee. To mark the blood vessels, vascular perfusion was performed using a green pigment-based contrast agent, following methods described in [3]. Labelling of the bone was then performed using an alizarin red staining protocol modified from [4]. Alizarin red is a water soluble dye that is used as a marker of extracellular matrix mineralization as it identifies calcium deposition. Following overnight paraformaldehyde-fixation, the tissue was washed, stained with alizarin, and then run through an ascending potassium hydroxide:glycerol series. The major challenge in imaging thick biological specimens is light scattering; clearing methods seek to better match the refractive indices and improve the penetration of light into tissues without compromising three-dimensional structure. Glycerol is a water-soluble optical tissue clearing agent known to be compatible with alizarin whole-mount staining. Cleared tissues were digitally imaged in whole mount using a Bausch and Lomb stereomicroscope or a Nikon Labophot 2 compound microscope. To estimate changes in vascular density during regeneration, random fields of view within the regenerated tail were recorded under the same imaging conditions and analyzed using ImageJ [5]. Images were 
segmented to discriminate vessels from background via manual thresholding by pixel intensity to determine the mean areal density of the vasculature within the regenerated tissue.

The contrast agent marking the vasculature persisted through the tissue processing required for the alizarin labeling of calcium deposits, allowing simultaneous imaging of both the regenerating microvasculature and bone. As expected, detectable vasculature was scant within the regenerating tissue in the first week, but preliminary results indicate that vascular density increased dramatically in the second week following amputation (Fig. 1A). With dual labeling, the perfused caudal artery, as well as an extensive microvascular network, were visible in concert with the caudal vertebrae (Fig. 1B). The tissue processing techniques presented here provide a technical foundation for subsequent investigations of the interrelationships between angiogenesis and osteogenesis within a profoundly regenerative environment. It is hoped that insights from a model system capable of appendage regeneration will ultimately provide strategies for the development of future regenerative therapies targeting angiogenesis and osteogenesis [6].

\section{References:}

[1] L Zhang et al. J Mater Sci Mater Med 25 (2014), p. 1165

[2] E Shipiani et al. J Bone Miner Res 24 (2009), p. 1347

[3] R Montoro and R Dickie. MethodsX 4 (2017), p. 265

[4] M Walker and C Kimmel. Biotech Histochem 82 (2007), p. 23

[5] C Schneider, W Rasband and K Eliceiri. Nature Methods 9 (2012), p. 671

[6] The authors acknowledge the Ambystoma Genetic Stock Center, which is funded by NIH grant P40OD019794, as the source of the animals.
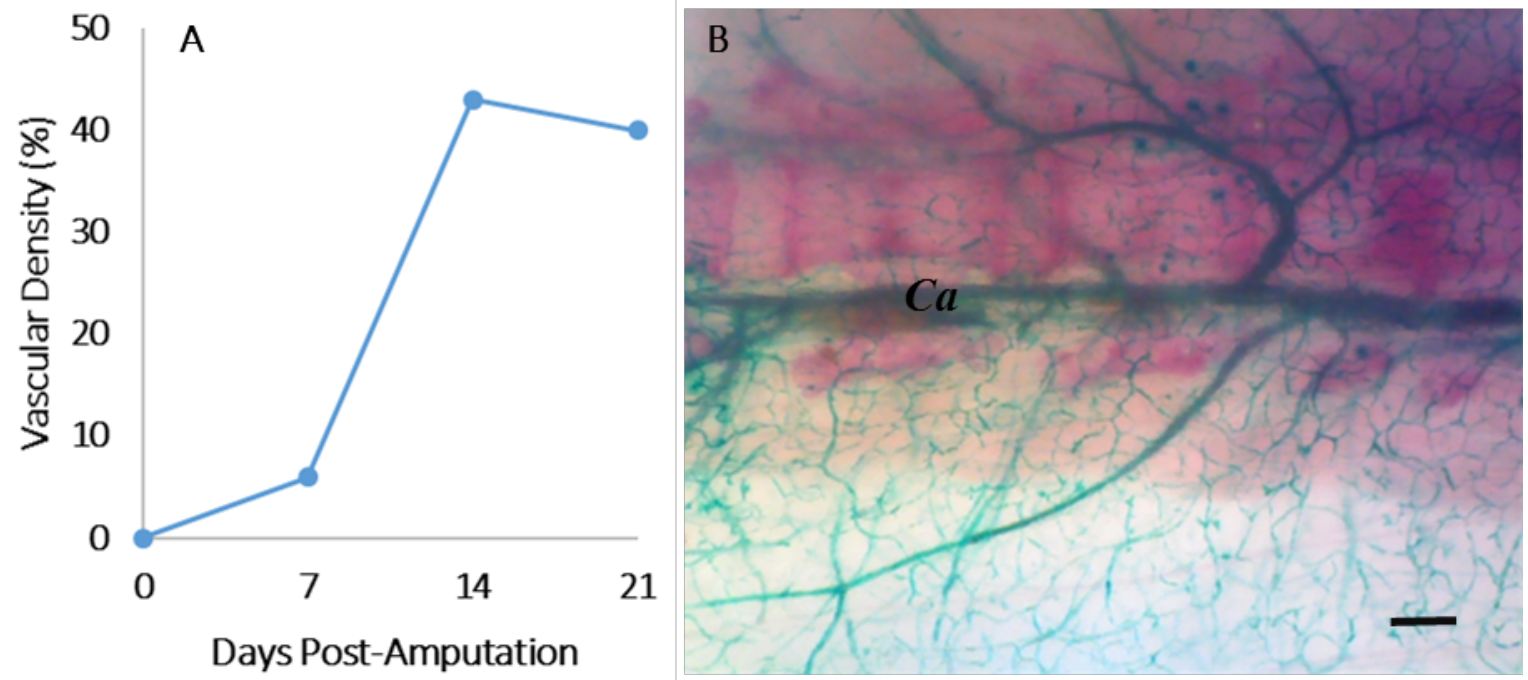

Figure 1. (A) Quantification of the perfused vessels within the regenerating tail suggested a dramatic increase in vascular density between the first and second week post-amputation. (B) Whole mount lateral view of regenerated tail tissue 42 days post-amputation, showing alizarin-labeled caudal vertebrae (pink) within the context of the perfused vessels (green). The caudal artery $(\mathrm{Ca})$ as well as a well-developed microvascular network are visible. Scale bar $=500 \mu \mathrm{m}$. 